\title{
Spin-orbit coupling in complexes of toluene with rare gas atoms
}

\author{
Thomas A. A. Oliver and Peter R. Taylor ${ }^{*}$ \\ Department of Chemistry, University of Warwick, Coventry CV4 7AL, United Kingdom \\ Richard J. Doyl $\AA^{\dagger}$ \\ Department of Chemistry, University of Durham, Durham DH1 3LE, United Kingdom \\ Stuart R. Mackenzi® \\ Department of Chemistry, University of Cambridge, Cambridge, United Kingdom
}

(Dated: April 24, 2007)

\begin{abstract}
The potential energy surfaces (PESs) and $S_{1}-T_{1}$ spin-orbit coupling matrix element (SOCME) surfaces are investigated for the toluene- $\mathrm{X}$ weakly-bound clusters $(\mathrm{X}=\mathrm{Ne}$, Ar, and $\mathrm{Kr})$. Calculations of the vibrational wave functions using a one-dimensional stretch model are presented and used to determine vibrationally-averaged values of the SOCMEs. Our ab initio theoretical results compare well with intersystem crossing rates derived from recent experimental fluorescence lifetime data [R. J. Doyle, E. S. J. Love, R. Da Campo, S. R. Mackenzie, J. Chem. Phys. 122, 194315]. Vibrational averaging is shown to change the absolute magnitude of the calculated SOCMEs, but the ratio between them remains very similar to that of the single-point values calculated at the minima of the PESs.
\end{abstract}

\section{INTRODUCTION}

The nonradiative decay of electronically excited molecules and clusters is a topic of considerable current interest in the fields of molecular properties and reaction dynamics. ${ }^{1 / 3}$ It is well known that the decay of excited states to other states of different multiplicity can occur via spin-orbit coupling, and the magnitude of this coupling can be highly sensitive to the local environment. Such effects have long been known in solution, where different solvents can lead to substantial changes in fluorescence lifetimes. In this context the phenomenon is known as the external heavy atom effect! 4

Several studies have shown that in isolated and weaklybound molecular clusters, the nearby presence of a single rare gas atom can greatly influence the decay dynamics of a molecule's excited state, by altering the magnitude of the spin-orbit coupling. Such a microscopic external heavy atom effect has been observed experimentally in rare gas van der Waals clusters of aromatic molecules including tetracene ${ }^{[5] 6}$ pentacene,$\frac{7}{11}$ octatetraene, ${ }^{8}$ azulene,, 9 substituted anthracenes, $10 / 11$ para-difluorobenzene $\frac{12}{12}$ and toluene ${ }^{13}$.

Calculations of external heavy atom effects in clusters range from approximate treatments using the atomic spin-orbit coupling values ${ }^{9}$ to more advanced methods treating the entire cluster. Non-relativistic ab initio calculations of spin-orbit coupling in weakly-bound clusters have mainly been restricted to small systems containing five or fewer atoms. For example, Minaev et al. have used response calculations to study phosphorescence rates in $\mathrm{C}_{2} \mathrm{H}_{2}-\mathrm{X}$ model systems $\left(\mathrm{X}=\mathrm{F}^{-}, \mathrm{Cl}^{-}\right.$, $\mathrm{Br}^{-}, \mathrm{HCl}$, and $\left.\mathrm{Ar}\right){ }^{14}$ An example of calculations treating larger systems is our previous experimental and theoretical study of para-difluorobenzene (pDFB) clustered with rare gas atoms, ${ }^{12}$ in which we calculated vibrationally averaged singlet-triplet spin-orbit coupling matrix elements
(SOCMEs) using a linear response approach.

In this paper we show that modern ab initio techniques can account quantitatively for the trend in lifetimes of the $S_{1}$ state of the toluene- $\mathrm{X}(\mathrm{X}=\mathrm{Ne}, \mathrm{Ar}, \mathrm{Kr})$ clusters with increasingly heavy cluster partner. We make use of multiconfigurational linear response theory, which enables the direct calculation of the singlet-triplet coupling matrix element without the explicit calculation of the triplet state wave function. We map out the magnitude of the coupling matrix element over a range of cluster geometries commensurate with the global minimum on the calculated intermolecular potential energy surface. Furthermore, we investigate the $S_{1}$ state potential energy surfaces of these systems and calculate vibrational wave functions for the relative motion of the cluster components in a restricted model. The vibrational averaging is shown to change slightly the absolute magnitude of the calculated SOCMEs, but the ratio between them remains almost identical to that of the single-point values calculated at the minima of the PESs. Both of the calculated ratios agree with the corresponding experimental ratio, within the reported uncertainties.

\section{POTENTIAL ENERGY CALCULATIONS}

We have performed electronic structure calculations on the toluene-rare gas systems, with the aim of determining a suitable model of the interaction potential for use in the calculation of vibrational levels. In our earlier work on the higher symmetry pDFB-rare gas systems, 12 we employed a simple representation where the motion of the rare gas atom was restricted to a line perpendicular to the aromatic ring and passing through its centre. Such a model may be considered less appropriate for the toluene-rare gas clusters, because toluene is lower in symmetry than pDFB, and the minimum-energy path 
along the potential surface deviates from the normal to the ring (hereafter termed the $R$-axis). However, as will be made clear below, the toluene-rare gas potentials are determined in this work to be roughly symmetric around the $R$-axis, indicating that the one-dimensional stretch model is in fact suitable as a first approximation.

The equilibrium geometry of the isolated toluene molecule in the excited $S_{1}$ state was first optimised, and then held fixed throughout the potential energy calculations. The justification for this is that the experiments with which we wish to make comparison were performed on the vibrationless $S_{1}$ levels 13 Also, the relatively low-frequency van der Waals modes of interest are not expected to greatly perturb the toluene geometry. The complete-active-space self-consistent field (CASSCF) method ${ }^{15}$ with six electrons in the $\pi$ space of six active orbitals, and the aug-cc-pVDZ basis set, 16 18 was used for the optimisation. The resulting geometry is detailed in Table I. The vibrational frequencies for the optimised structure are real, and the methyl group hydrogens are staggered with respect to the ring. For comparison the table also lists the geometry described by East et $a l ! 19$, who combined experimental fluorescence data with calculated CASSCF /6-31G(d,p) results in a least-squares fit (referred to as LSQ II).

The cluster potential energy data was generated using the MOLCAS package ${ }^{20}$ with the CASPT2 method and the aug-cc-pVDZ basis set. Core orbitals on both toluene and the noble gas atoms were frozen and only valence electrons correlated for all CASPT2 calculations. The inclusion of core-valence correlation would be expected to increase the depth of the interaction potential somewhat for the Ar and Kr clusters, since it will increase the calculated polarizability. At the present level of accuracy this can be ignored. Counterpoise calculations were carried out at each point to mitigate against basis set superposition error (BSSE) using the method of Boys and Bernardi21. Intruder state problems were encountered with the CASPT2 calculations and resolved by using an imaginary level shift as devised by Forsberg and Malmqvist 22 .

The $R, x, y$ coordinate system used in this work for specifying the location of the rare gas nucleus relative to the toluene molecules is depicted in Fig. 1. At its equilibrium geometry, toluene assumes the $C_{\mathrm{s}}$ point group and the potential is symmetric on each side of the symmetry plane. The origin of the coordinate system was chosen as the mid-point between the $\mathrm{C} 1$ and $\mathrm{C} 4$ atoms on the $C_{\mathrm{s}}$ axis. The variation in the potential along $x$ is mappedout in Fig. 2 at fixed $R=3.374 \AA$. It can be seen that the potential is almost symmetric about $x=0$. As expected, the potential is similar to those calculated for other aromatic ring-rare gas systems, where global potential minima are found above the center of the rings 23,27 Our potential curves along the $R$ axis are shown in Fig. 3 . These curves were used in the approximate treatment of the vibrational averaging in section IV.
TABLE I: Optimised geometry of the $S_{1}$ state toluene molecule using CASSCF/aug-cc-pVDZ, compared with "LSQ II" data from Ref. [19] where their torsional angle $\rho=90^{\circ}$ (i.e., the staggered conformation). $R_{\mathrm{AB}}$ refers to the distance in $\AA$ between nuclei of $\mathrm{A}$ and $\mathrm{B}$, and $\angle \mathrm{ABC}$ refers to the angle in degrees between the $\mathrm{B} \rightarrow \mathrm{A}$ and $\mathrm{B} \rightarrow \mathrm{C}$ vectors.

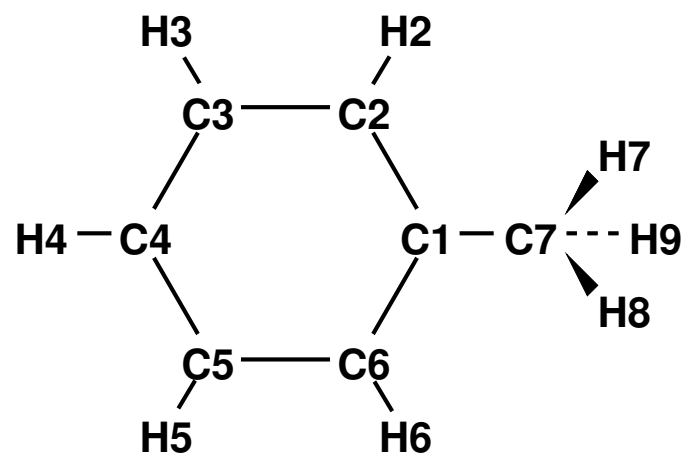

\begin{tabular}{lll}
\hline \hline Property & CASSCF & LSQ II \\
\hline$R_{C 1, C 7}$ & 1.504 & 1.499 \\
$R_{C 1, C 2}$ & 1.441 & 1.439 \\
$R_{C 2, C 3}$ & 1.436 & - \\
$R_{C 3, C 4}$ & 1.434 & 1.432 \\
$R_{C 2, H 2}$ & 1.079 & 1.084 \\
$R_{C 3, H 3}$ & 1.079 & 1.084 \\
$R_{C 4, H 4}$ & 1.079 & 1.084 \\
$R_{C 7, H 9}$ & 1.094 & 1.101 \\
$R_{C 7, H 7}$ & 1.090 & - \\
$\angle_{C 2, C 1, C 6}$ & 119 & 119 \\
$\angle_{C 1, C 2, C 3}$ & 121 & - \\
$\angle_{C 2, C 3, C 4}$ & 120 & - \\
$\angle_{C 3, C 4, C 5}$ & 120 & 120 \\
\hline \hline
\end{tabular}

\section{SPIN-ORBIT COUPLING MATRIX ELEMENTS}

In order to determine the interaction between the singlet $\left|S_{1}\right\rangle$ and triplet $\left|T_{1}\right\rangle$ electronic states for the toluene$\mathrm{X}$ systems, calculations of the appropriate matrix elements of the spin-orbit operator were performed. We have calculated these matrix elements as residues of a triplet linear response function 28 , with a multiconfigurational reference $\left|S_{1}\right\rangle$ wave function,

$$
\left\langle S_{1}\left|H_{\mathrm{so}}\right| T_{1}\right\rangle=\lim _{E \rightarrow E_{T_{1} S_{1}}}\left(E-E_{T_{1} S_{1}}\right)\left\langle\left\langle H_{\mathrm{so}} ; V(E)\right\rangle\right\rangle_{E},
$$

where $V$ is an excitation operator and $E_{T_{1} S_{1}}$ is the energy gap between the singlet and triplet states. $H_{\text {so }}$ is the spin-orbit operator, for which we employed the meanfield formulation of Heß et al. $[29]$. The $S_{1}$ CASSCF state was calculated with a $(6 / 6)$ pi electron active space and the aug-cc-pVDZ basis set. These multiconfigurational linear response (MCLR) calculations were performed using the DALTON quantum chemistry program $\frac{30}{3}$ We 


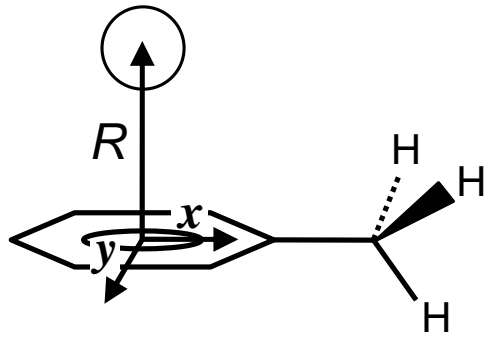

FIG. 1: Schematic diagram defining the coordinate system used in this work.

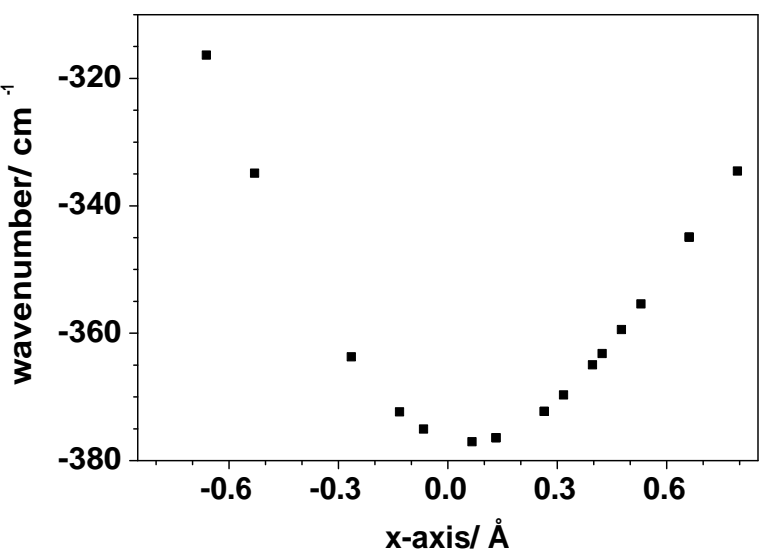

FIG. 2: A cut through the toluene-Ar $S_{1}$ potential surface, for $y=0$ and $R=3.374 \AA$.

should perhaps note that since the MCLR calculations are performed in a determinantal basis, without explicit spin adaptation, it is occasionally the case that the $S_{0}$ state appears as the lowest energy "excited state", but the desired $T_{1}$ state is easily obtained by taking an extra root of the MCLR equations.

Matrix elements were calculated for a range of raregas coordinates in the $x-R$ plane. Coordinates for the position of the rare gas atom with $+R$ are not equivalent to those with $-R$ (i.e., for the rare gas atom reflected in the plane of the ring), due to the presence of the methyl group. However, it may be expected that the differences in the matrix elements for $\pm R$ are negligibly small in the region around the center of the ring. In order to test this assumption, calculations were performed for toluene- $\mathrm{Ar}$ covering both sides of the ring. As can be seen in Fig. 4 the variation between the values on each side, around $x=0$, is small and all further calculations were performed on one side only (the side with $R>0$ in Fig. 11. The origin of the asymmetry in the SOCMEs further away from $x=0$ may involve the slight non-planarity of the ring due to the methyl group.

Contour plots for the matrix elements of toluene-Ne

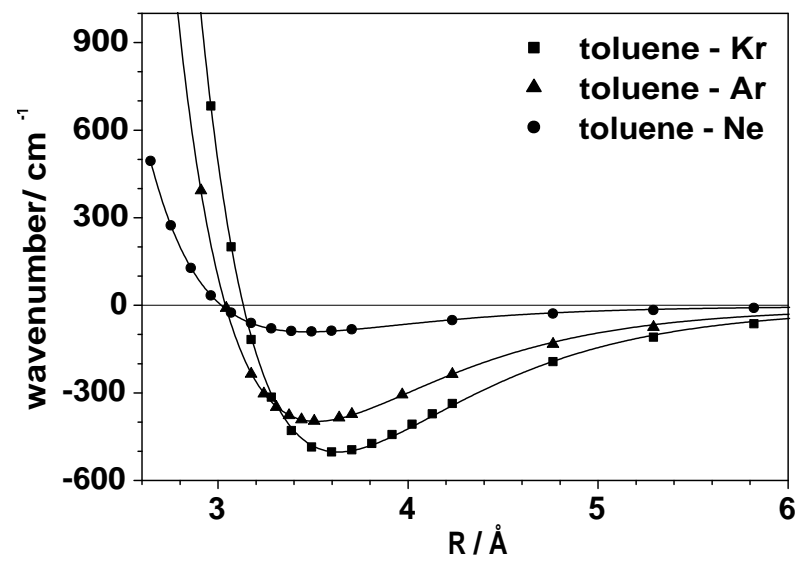

FIG. 3: $S_{1}$ state potential energy curves for toluene-X $(\mathrm{X}=\mathrm{Ne}, \mathrm{Ar}, \mathrm{Kr})$ shown as a function of $R$ for $\mathrm{x}=\mathrm{y}=0$.

and toluene- $\mathrm{Kr}$ are shown in Figs. 5 and 6 respectively. For comparison, the matrix elements for all three clusters are plotted along the $R$-axis in Fig.7. In the limit of large $R$, the SOCMEs converge to the same asymptotic value of $0.1215 \mathrm{~cm}^{-1}$. This wavenumber corresponds in principle to the SOCME for toluene, unperturbed by the presence of a cluster partner. However, this value is not expected to be numerically accurate because of the difficulty in calculating SOCMEs of such small magnitude using the current level of theory.

\section{VIBRATIONAL AVERAGING AND COMPARISON WITH EXPERIMENT}

Experimentally, the fluorescence lifetimes of the toluene-rare gas atom $S_{1}$ states have been shown to vary considerably from 44 ns for toluene-Ar to 4 ns for toluene-Kr, in comparison to 84 ns for the uncomplexed toluene molecule. ${ }^{13}$ Such lifetimes are longer than the equivalents for para-difluorobenzene. ${ }^{12}$ It has previously been argued that the explanation for this trend lies in the increase in SOC between $S_{1}$ and $T_{1}$ with the presence of more massive rare gas atoms in the clusters. ${ }^{13}$ If this is the case, the ratio of the experimental fluorescence decay rates should agree with the ratio of the calculated SOCMEs. One simple (semiclassical) way to make such a comparison is to take the values of the SOC matrix elements for the equilibrium geometries, i.e., for the $R$ coordinate where the potential curves are at their minimum. One step up from this semiclassical approach is to allow the rare gas atom to move along the $R$ axis relative to toluene, and determine the energy and wavefunction for the lowest-energy $(\mathrm{v}=0)$ quantum level of this onedimensional model. The SOCME can then be averaged over this vibrational motion. In other words, the expectation value of the SOCME is determined for the lowestenergy vibration of $S_{1}$. The Hamiltonian for the model 

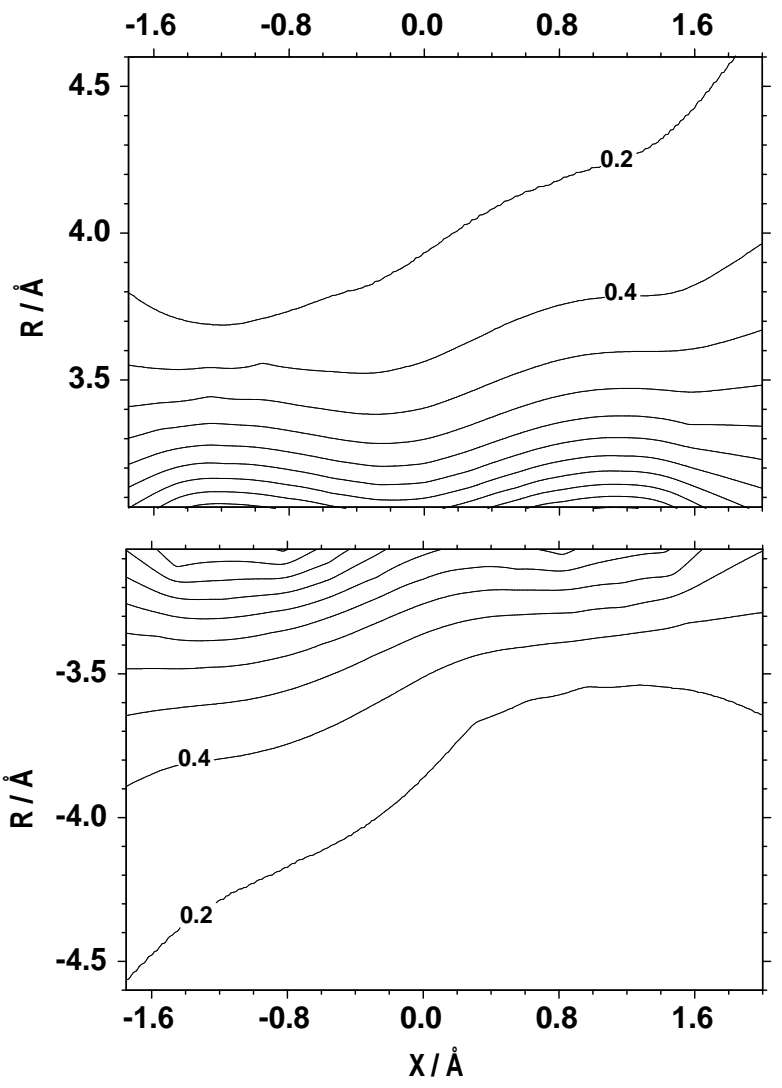

FIG. 4: Contour plot of the SOCME surface for toluene-Ar. The contours are equally spaced in steps of $0.2 \mathrm{~cm}^{-1}$.

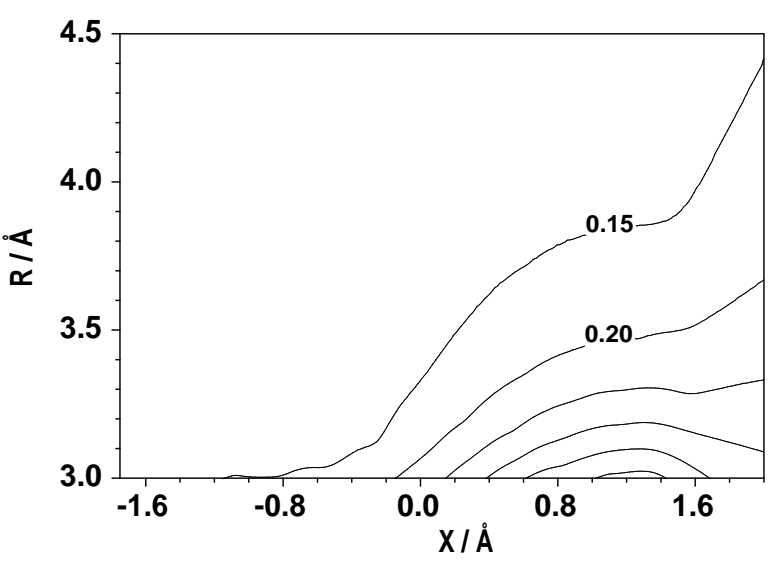

FIG. 5: Contour plot of the SOCME surface for toluene-Ne. The contours are equally spaced in steps of $0.05 \mathrm{~cm}^{-1}$.

one-dimensional stretch systems can be written as

$$
\hat{\mathrm{H}}=\frac{\hbar^{2}}{2 \mu}\left(\frac{\mathrm{d}^{2}}{\mathrm{~d} R^{\prime 2}}\right)+\hat{\mathrm{V}}\left(R^{\prime}\right),
$$

where $\mu$ is the reduced mass of the toluene- $\mathrm{X}$ system, $R^{\prime}$ is the distance between the rare gas atom and the center-of-mass of toluene, and $\hat{V}$ is the potential energy operator. The vibrational energies and wave

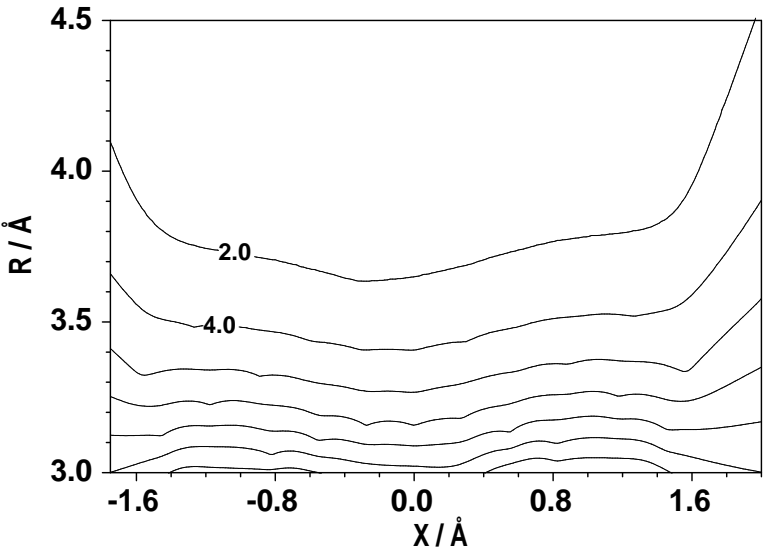

FIG. 6: Contour plot of the SOCME surface for toluene-Kr. The contours are equally spaced in steps of $2 \mathrm{~cm}^{-1}$.

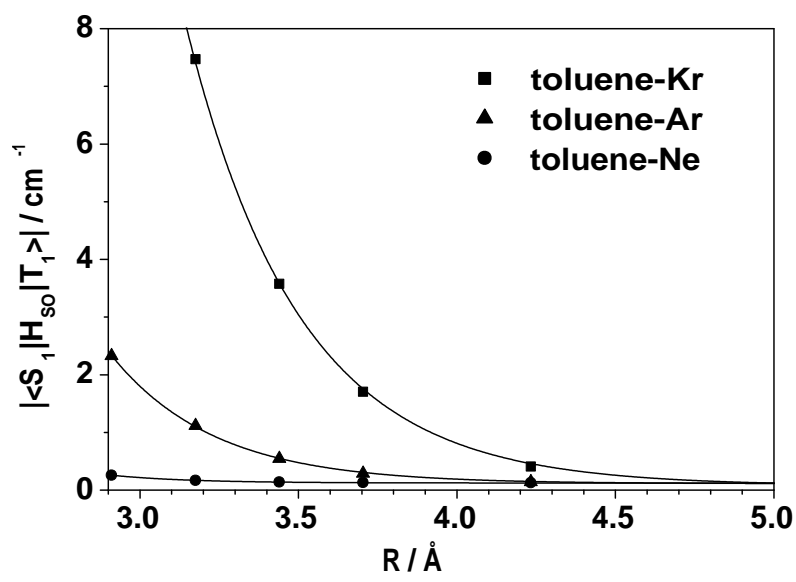

FIG. 7: Cuts through the SOCME surfaces at $x=y=0$. The symbols indicate the calculated data points, whilst the solid lines are least-squares fits to exponential decays.

functions were generated by diagonalising $\hat{H}$ in a basis of sinc-DVR (Discrete Variable Representation) ${ }^{31}$ functions. The overlap integral of the square of the groundstate $(\mathrm{v}=0)$ wave function with the calculated SOCME data provides the vibrationally-averaged value of the SOCME. A comparison of the vibrationally-averaged, semiclassical and experimental data is presented in Table II] The experimental data is presented in terms of $\Gamma_{\text {HA }}$ (Heavy Atom) values, defined as the difference in the nonradiative decay rate between the clustered and unclustered molecule $\mathrm{e}^{12 \mid 13}$. This parameter reflects the experimentally-determined increase in the rate of spinorbit coupling upon clustering $12 \mid 13$. According to Fermi's golden rule, the $T_{1} \leftarrow S_{1}$ transition rate is proportional to the square of the SOCME. In order to make a comparison between our theoretical calculations and the experimental $\Gamma_{\text {HA }}$ values, it is necessary to determine the squares of the calculated SOCMEs and subtract the contribution from the bare toluene molecule. This gives us a theoretical prediction for the relative increases in the transi- 
tion rate due to clustering. The ratio of these adjusted squared SOCMEs can then be compared with the ratio of the experimental $\Gamma_{\text {HA }}$ values. In Table II, the squared SOCMEs have been adjusted as described above by subtracting their asymptotic values, and the appropriate ratios are compared. The theoretical ratios for both the single-point and vibrationally-averaged values fall well within the uncertainties in the experimental ratio. The vibrational averaging proceedure alters the magnitude of the SOCMEs to some extent: for the toluene-Ne complex the value is slightly reduced, whereas for the other two species the SOCME is increased. However, the ratio of the squares of the vibrationally-averaged SOCMEs is very similar to that for the single-point values and both fall well within the range of experimental uncertainty.

In conclusion, the calculated SOCMEs for clusters of toluene with rare-gas atoms agree well when compared to the experimental data at its current level of accuracy. A simple vibrational-averaging proceedure slightly alters the absolute magnitude of the SOCMEs, but the ratio between them remains very similar to the ratio of the SOCMEs calculated at the minima of the potential surfaces.

\section{SUMMARY}

We have presented calculations of potential energy surfaces and SOCMEs for a range of toluene-rare gas atom clusters, and have determined vibrationally-averaged values for the SOCMEs. These compare very well with experimentally-derived fluorescence lifetime data, supporting the explanation that spin-orbit coupling from the $S_{1}$ to the $T_{1}$ state is responsible for the trends in lifetimes observed.

\section{ACKNOWLEDGEMENTS}

The authors thank the Nuffield Foundation for funding TAAO's Undergraduate Research Bursary. PRT wishes to thank the Wolfson Foundation for support through the Royal Society. Furthermore, the UK Engineering and Physical Sciences Research Council (EPSRC) are thanked for funding SRM's Advanced Research Fellowship, and for the grant which originally funded the experimental work on toluene clusters (GR/R36367/01).
* Electronic address: P.R.Taylor@warwick.ac.uk

† Electronic address: richard.j.doyle@gmail.com

¥ Electronic address: srm49@cam.ac.uk

1 T. E. Dermota, Q. Zhong, and A. W. Castleman, Jr., Chem. Rev. 104, 1861 (2004).

2 O. David, C. Dedonder-Lardeux, and C. Jouvet, Int. Rev. Phys. Chem. 21, 499 (2002).

3 A. L. Sobolewski, W. Domcke, C. Dedonder-Lardeux, and C. Jouvet, Phys. Chem. Chem. Phys. 4, 1093 (2002).

${ }^{4}$ S. P. McGlynn, T. Azumi, and M. Kinoshita, Molecular Spectroscopy of the Triplet State (Prentice-Hall, Englewood Cliffs, NJ, 1969).

5 A. Amirav, U. Even, and J. Jortner, Chem. Phys. Lett. 67, 9 (1979).

6 A. Amirav, U. Even, and J. Jortner, J. Chem. Phys. 75, 2489 (1981).

7 A. Amirav, U. Even, and J. Jortner, J. Phys. Chem. 85, 309 (1981).

8 T.-S. Kim, Y. S. Choi, and K. Yoshihara, Chem. Phys. Lett. 247, 541 (1995).

9 O. K. Abou-Zied, D. R. M. Demmer, S. C. Wallace, and R. P. Steer, Chem. Phys. Lett. 266, 75 (1997).

10 L. C. T. Shoute, S. Hirayama, and R. P. Steer, Chem. Phys. Lett. 338, 123 (2001).

11 S. Hirayama, F. Tanaka, and K. Shobatake, Chem. Phys. Lett. 153, 112 (1988).

12 R. J. Doyle, R. Da Campo, P. R. Taylor, and S. R. Mackenzie, J. Chem. Phys. 121, 835 (2004).

13 R. J. Doyle, E. S. J. Love, R. Da Campo, and S. R. Mackenzie, J. Chem. Phys. 122, 194315 (2005).

14 B. F. Minaev, S. Knuts, and H. Ågren, Chem. Phys. 181, 14 (1994).

15 P. E. M. Siegbahn, J. Almlöf, A. Heiberg, and B. O. Roos, J. Chem. Phys. 74, 2384 (1981).
16 T. H. Dunning Jr., J. Chem. Phys. 90, 1007 (1989).

17 R. A. Kendall, T. H. Dunning Jr., and R. J. Harrison, J. Chem. Phys. 96, 6796 (1992).

18 D. E. Woon and T. H. Dunning Jr., J. Chem. Phys. 100 2975 (1994).

19 A. L. East, E. C. Lim, P. Jenson, I. Dechene, M. Z. Zgierski, W. Siebrand, and P. R. Bunker, J. Chem. Phys. 112, 167 (2000).

20 G. Karlström, R. Lindh, P.-Å. Malmqvist, B. O. Roos, U. Ryde, V. Veryazov, P.-O. Widmark, M. Cossi, B. Schimmelpfennig, P. Neogrady, et al., Comput. Mater. Sci. 28, $222(2003)$.

21 S. F. Boys and F. Bernardi, Mol. Phys. 19, 553 (1970).

22 N. Forsberg and P. Malmqvist, Chem. Phys. Lett. 247, 196 (1997).

23 J. Makarewicz, J. Chem. Phys. 124, 044310 (2006).

24 H. Koch, B. Fernandez, and J. Makarewicz, J. Chem. Phys. 111, 198 (1999).

25 B. Fernandez, H. Koch, and J. Makarewicz, J. Chem. Phys. 111, 5922 (1999).

26 J. Makarewicz, J. Chem. Phys. 122, 114312 (2005).

27 C. R. Munteanu, J. L. Cacheiro, B. Fernandez, and J. Makarewicz, J. Chem. Phys. 121, 1390 (2004).

28 J. Olsen, D. L. Yeager, and P. Jørgensen, J. Chem. Phys. 91, 381 (1989).

29 B. A. Heß, C. M. Marian, U. Wahlgren, and O. Gropen, Chem. Phys. Lett. 251, 365 (1996).

30 Dalton, a molecular electronic structure program, release 1.2 (2001), see http://www.kjemi.uio.no/software/dalton/dalton.html.

31 S. E. Choi and J. C. Light, J. Chem. Phys. 92, 2129 (1990). 
TABLE II: Calculated SOCMEs and experimental decay rates for $S_{1}$ cluster states. The experimental ratio is given as the ratio of $\Gamma_{\mathrm{HA}}$ values from Ref. 13

\begin{tabular}{l|l|l|l|l|l}
\hline \hline \multirow{2}{*}{ Species } & \multicolumn{2}{|c|}{$\left|\left\langle S_{1}\left|H_{\mathrm{so}}\right| T_{1}\right\rangle\right|^{2}$} & \multicolumn{3}{c}{ Ratio } \\
\cline { 2 - 6 } & value at $\mathrm{R}_{e}$ & vibrationally-averaged & value at $\mathrm{R}_{e}$ & vibrationally-averaged & Experimental ratio \\
\hline toluene- $\mathrm{Ne}$ & $3.94 \times 10^{-3}$ & $3.87 \times 10^{-3}$ & $2.04 \times 10^{-2}$ & $1.93 \times 10^{-2}$ & $0.08 \pm 0.1$ \\
toluene- $\mathrm{Ar}$ & 0.193 & 0.200 & 1.00 & 1.00 & 1.00 \\
toluene- $\mathrm{Kr}$ & 4.55 & 4.70 & 23.5 & 23.5 & $22.6 \pm 12$ \\
\hline \hline
\end{tabular}

